\title{
Orientation Distribution and Mean Width Determination in micro X-Ray CT Images of Fibrous Materials
}

\author{
John Chiverton ${ }^{1}$, Alexander Kao ${ }^{1}$, Gianluca Tozzi ${ }^{1}$, Marta Roldo ${ }^{2}$ \\ 1. School of Engineering, University of Portsmouth, Portsmouth, UK, PO1 3DJ, \\ Email: john.chiverton@port.ac.uk, gianluca.tozzi@port.ac.uk \\ 2. School of Pharmacy and Institute of Biomedical and Biomolecular Science, \\ University of Portsmouth, UK, PO1 2DT, Email: marta.roldo@port.ac.uk
}

Keywords: 3D Image Processing, XCT, Fibres

\begin{abstract}
Fibrous nano-materials can be imaged using high resolution $\mathrm{X}$-ray computer tomography (XCT). Some important parameters that are often manually estimated from this imaging data include the orientation distribution and the thickness of the fibres. Automation of this process is hampered by the close proximity of the fibres even with sub-micron voxel sizes. An automated sampling methodology has therefore been developed that can detect points in the imaging data where fibres are present and well separated. Polycaprolactone (PCL) electrospun fibrous material was prepared and imaged with a Zeiss Xradia Versa 510 Microtomography XCT. Automated measurements were then determined and further summarised using a single parameter measure.
\end{abstract}

\section{Introduction}

Man made fibrous nano-materials such as electrospun materials can be used for many applications such as for wound dressings, filtration, biosensors, tissue engineering, drug delivery [1]. Imaging of these man made nano-materials requires high resolution imaging techniques such as high resolution $\mathrm{X}$-ray computer tomography (XCT). The work presented here could be considered to having some application to these areas.

Fibres are often modelled as tubes that can be detected using Eigen-analysis of the Hessian of the 3D imaging data. This approach was proposed by Sato et al. [2] and found repeated use over the years [3], [4], [5]. Other works may rely on fibre tracing in conjunction with e.g. the use of a distance transform such as Huang et al. [6]. More recently machine learning centric approaches have attracted some interest such as the work by Kritrungrotsakul et al. [7]. Difficulties such as poor interslice sampling can also be dealt with using robust fitting algorithms such as the work by Chiverton et al. [8]

Some important parameters that are often estimated using semi-manual interaction with image processing tools include deriving the orientation distribution and manually assisted measurement of the thickness of the fibres [9], [10].
An aspect not directly considered in works such as the ones mentioned above is regarding the Partial Volume (PV) effect [11]. Any image or signal acquisition process will be associated with a finite sampling resolution which manifests itself in the form of blurred image intensities known as PV affected voxels. Measurements of structures of interest such as the diameters of fibres can therefore be problematic. This is particularly true if the spread of the image intensities is wide in relation to the structure of interest which is often the case with imaging of fibre based materials and tissues. Another point of difficulty can arise when the fibres have close proximity in relation to the spread of the intensities, which again is a likely scenario. Imaging artifacts may also result in inhomogeneities in the image intensities [12] which may be more of a problem for denser materials.

Automation of these image summarization techniques is hampered by close proximity of fibres even when using image data acquired with sub-micron voxel sizes. We have therefore devised an automated sampling methodology that can detect points in the imaging data where fibres are present and which are well separated from neighbouring fibres. These points provide optimal locations to perform orientation and thickness measurements and thus enable automated measurements of

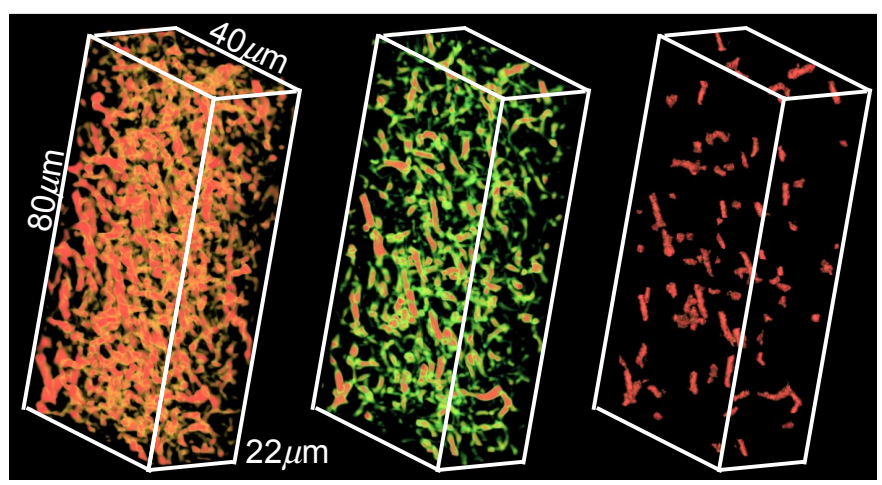

Figure 1: From left to right: (a) Volume rendering of Region of Interest (ROI) of PCL fibre volume consisting of $55 \times 100 \times$ 200 voxels, each of dimension $0.4^{3} \mu \mathrm{m}^{3}$ covering a region in size $70400 \mu \mathrm{m}^{3}$; (b) volume rendering after fibre enhancement process of (a); (c) isolated fibres from thresholding (b). 
these variables. Imaging data of fibrous materials is collected using our Zeiss Xradia Versa 510 Microtomography X-Ray Computer Tomography (XCT) system at the University of Portsmouth's Zeiss Global Centre. Diameter measurements are automatically obtained which match well with the expected thickness of the manufactured material. The orientation distribution is also calculated, visualised and further summarised using a single parameter measure together with the distribution of the fibres throughout the scanned volume.

\section{Methodology}

A 3D image volume $I$ is considered here to map 3D voxel positions $\boldsymbol{x}=\left(\begin{array}{lll}x & y & z\end{array}\right)^{T} \in \Omega$ to scalar image intensities, i.e. $I: \Omega \rightarrow \Re$ where $\Omega \subset \Re^{3}$. The Hessian matrix $\mathcal{H}_{\eta}$ at scale $\eta$ of second derivatives of $I$ can be computed for every voxel position with

$$
\mathcal{H}_{\eta}(\boldsymbol{x})=\left(\begin{array}{ccc}
\frac{\partial I_{\eta}(\boldsymbol{x})}{\partial x \partial x} & \frac{\partial I_{\eta}(\boldsymbol{x})}{\partial x \partial y} & \frac{\partial I_{\eta}(\boldsymbol{x})}{\partial x \partial z} \\
\frac{\partial I_{\eta}(\boldsymbol{x})}{\partial y \partial x} & \frac{\partial I_{\eta}(\boldsymbol{x})}{\partial y \partial y} & \frac{\partial I_{\eta}(\boldsymbol{x})}{\partial x \partial z} \\
\frac{\partial I_{\eta}(\boldsymbol{x})}{\partial z \partial x} & \frac{\partial I_{\eta}(\boldsymbol{x})}{\partial z \partial y} & \frac{\partial I_{\eta}(\boldsymbol{x})}{\partial z \partial z}
\end{array}\right),
$$

where $I_{\eta}(\boldsymbol{x})$ is the image data convolved with a Gaussian with width $\eta$ at point $\boldsymbol{x}$. The scale, in particular in conjunction with a Gaussian smoothing kernel is important because the derivative is a discrete approximation. The second derivatives of the smoothed image data, convolved with a Gaussian kernel can be approximated with the convolution of the image data with the analytically derived second derivative of a Gaussian due to the linear space invariant property of this combined process. The scale notation is not included any further for the sake of brevity. The eigenvalues $\lambda_{1, \boldsymbol{x}}, \lambda_{2, \boldsymbol{x}}$ and $\lambda_{3, \boldsymbol{x}}$ of $\mathcal{H}(\boldsymbol{x})$ can then be calculated, i.e. $\mathcal{H}(\boldsymbol{x})=U_{\boldsymbol{x}} \Lambda_{\boldsymbol{x}} U_{\boldsymbol{x}}^{\mathrm{T}}$ where $U_{\boldsymbol{x}}$ is an eigenvector matrix and $\Lambda_{\boldsymbol{x}}$ is the diagonal matrix of eigenvalues. It is well known that bright tubular structures can be detected by finding points where $\lambda_{3, \boldsymbol{x}} \approx 0, \lambda_{2, \boldsymbol{x}} \ll 0$ and $\lambda_{1, \boldsymbol{x}} \ll 0$ (see e.g. [2]). Tubular structures can then be enhanced with (from [2])

$$
V(\boldsymbol{x})=\left\{\begin{array}{cc}
0 & \text { if } \kappa<0 ; \\
\kappa \exp \left(-\frac{\lambda_{3, \boldsymbol{x}}^{2}}{2 \alpha \kappa}\right) & \text { elsewhere; }
\end{array}\right.
$$

where $\kappa=\min \left(-\lambda_{1, \boldsymbol{x}},-\lambda_{2, \boldsymbol{x}}\right)$. A binary map is created $B(\boldsymbol{x})$ taking a value of 1 for points that are good candidates for a fibre if $V(\boldsymbol{x})$ exceeds a threshold $\tau_{\eta}$, i.e.

$$
B(\boldsymbol{x})= \begin{cases}1 & \text { if } \quad V(\boldsymbol{x}) \geq \tau_{\eta} \\ 0 & \text { elsewhere }\end{cases}
$$

The threshold $\tau_{\eta}$ is dependent on the scale because the statistical properties of $V(\boldsymbol{x})$ vary dependent on the scale. The threshold is therefore made a function of the percentile of the statistical properties of $V(\boldsymbol{x})$, i.e.

$$
\tau_{\eta}=\mathrm{CDF}_{V}^{-1}(p=0.97)
$$

where $\mathrm{CDF}^{-1}$ is the inverse of the cumulative distribution function of $V(\boldsymbol{x})$. An illustration of the values of $\tau_{\eta}$ for a range of scales can be seen in Fig. 2.

Clusters of connected points in the binary map $B(\boldsymbol{x})$ are then identified where the $k^{\text {th }}$ cluster is given by $C_{k}=$

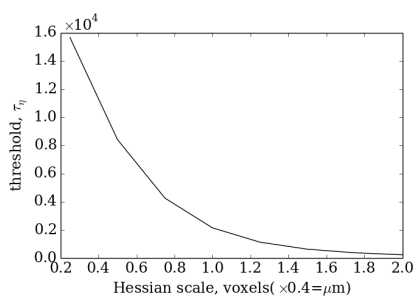

Figure 2: Illustration for a range of different scales showing how the threshold $\tau_{\eta}$ varies, used to create the binary map in (3).

$\left\{\boldsymbol{x}_{i} \mid(\exists j)\left[B\left(\boldsymbol{x}_{i}\right) \wedge B\left(\boldsymbol{x}_{j}\right) \wedge\left\langle\boldsymbol{x}_{i}, \boldsymbol{x}_{j}\right\rangle \leq d\right]\right\}$ for some threshold distance $d$ which is typically given by the voxel size on a single dimension scaled by $\sqrt{3}$ for 26 connectivity. The major and minor axes of each cluster are then computed via PCA which is performed for these clusters using the process described next.

Major and Minor Axis Determination. Initially the sample covariance matrix for each cluster is computed

$$
\mathbf{Q}_{k}=\frac{1}{\left|C_{k}\right|-1} \sum_{\forall \boldsymbol{x}_{i} \in C_{k}}\left(\boldsymbol{x}_{i}-\boldsymbol{\mu}_{k}\right)\left(\boldsymbol{x}_{i}-\boldsymbol{\mu}_{k}\right)^{\mathrm{T}}
$$

where $\boldsymbol{\mu}_{k}$ is the center of cluster $k$, i.e. the mean computed with $\boldsymbol{\mu}_{k}=\frac{1}{\left|C_{k}\right|} \sum_{\forall \boldsymbol{x}_{i} \in C_{k}} \boldsymbol{x}_{i}$.

PCA for each cluster is then computed via the eigenvector decomposition of each covariance matrix $\mathbf{Q}_{k}=R_{k} \Sigma_{k} R_{k}^{\mathrm{T}}$ where $R_{k}$ and $\Sigma_{k}$ are a matrix of eigenvectors and a matrix of eigenvalues respectively for the $k^{\text {th }}$ cluster of connected voxels. The eigenvectors indicate here candidate major and minor axes on which the eigenvalues describe the amount of spread. The major axis is the direction given by the eigenvector with the greatest eigenvalue, i.e. $\lambda_{1, k}$ where $\lambda_{1, k} \geq \lambda_{2, k} \geq \lambda_{3, k}$. A fibre would be expected to have the longest length along this axis; whilst the two eigenvectors corresponding to the two smaller eigenvalues $\lambda_{2, k}$ and $\lambda_{3, k}$ would be expected to form the minor axes of the fibre because they are orthogonal to the major axis. Due to the statistical nature of the aforementioned processes, it is likely that a number of candidate fibres may need to be rejected. This might be because of a number of reasons, such as clusters of high intensity points that may be somewhat fibre like but in actual fact are poor candidates for this sampling process. Another reason might be due to a fibre being highly irregular but still tubular and with high intensity. The above fibre enhancement stage may still identify those as candidate fibres but are likely to act as poor candidates for subsequent statistical analysis of fibre properties.

Fibre Candidacy. A further stage is therefore needed to determine if a fibre conforms to a number of requirements that would enable useful properties to be associated with an individual fibre. These requirements are: the two minor axis estimated diameters of the fibre should be approximately equal; and the two minor axis intensity profiles should be similar including maximum and minimum intensities. These two properties can be determined by traversing the two minor 

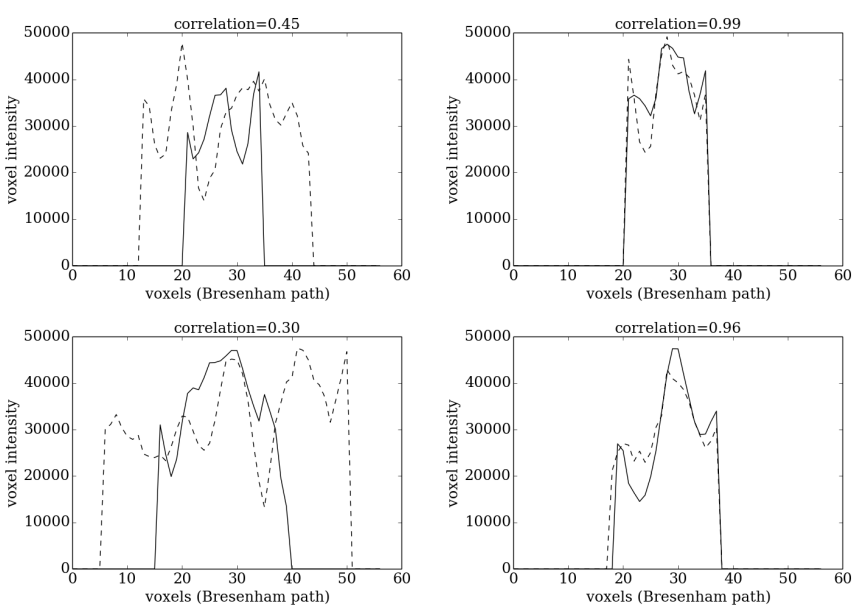

Figure 3: Exemplar minor axes (orthogonal) line profiles, illustrating line profiles with low correlation and line profiles with high correlation. The line profiles are taken along Bresenham paths [13], stepping through discrete voxel locations. The actual length is computed subsequently. Also note the variation in minima surrounding the central peak (maximum) values in each line profile.

axes through the mean point $\boldsymbol{\mu}_{k}$ as an indicator of the center of the fibre.

The similarity of the maximum $I_{2, k}^{\max }, I_{3, k}^{\max }$ and minimum values $I_{2, k}^{\min }, I_{3, k}^{\min }$ for the two minor axes $(2,3)$ for cluster $k$ can be calculated with

$$
\begin{gathered}
D_{k, \text { max }}=\frac{\left|2\left(I_{2, k}^{\max }-I_{3, k}^{\max }\right)\right|}{\left|\left(I_{2, k}^{\max }+I_{3, k}^{\max }\right)\right|} \text { and } \\
D_{k, \min }=\frac{\left|2\left(I_{2, k}^{\min }-I_{3, k}^{\min }\right)\right|}{\left|\left(I_{2, k}^{\min }+I_{3, k}^{\min }\right)\right|} .
\end{gathered}
$$

If the maximum similarity has a value below e.g. $D_{k, \max } \leq$ 0.02 then we consider it to be approximately equal as the maximum should coincide with the center of the fibre through which both axes traverse. However, the minimum value is likely to vary more widely so we found, by empirical experimentation that a value of $D_{k, \text { min }} \leq 0.2$ gave a strong indication of a fibre with some geometric properties that could prove useful in the statistical characterisation of the data.

A further test to determine a candidate fibre's minor axis properties is to compare the intensity profiles for the two minor axes. Correlation in the form of the sample correlation coefficient is used here to compare the line intensity profiles $I_{l, 2, k}$ and $I_{l, 3, k}$ where

$$
\rho_{k_{2,3}}=\frac{\sum_{l=0}^{L-1}\left(I_{l, 2, k}-\mu_{2, k}\right)\left(I_{l, 3, k}-\mu_{3, k}\right)}{(L-1) \eta_{2, k} \eta_{3, k}}
$$

where the line profiles have $L$ voxels and individual voxels, indexed by $l \in L$ are determined using Bresenham's line algorithm [13]. A high correlation e.g. $\rho_{k_{2,3}} \geq 0.9$ would indicate that the intensity line profiles correlate reasonably well which increases the possibility that the candidate fibre is correct. Some comparative line profiles can be seen in Fig. 3.
Fibre Diameter Determination. It might be expected that the diameter of a fibre could potentially be found by finding the point at which the intensity of the fibre drops down to a level comparable to the surrounding medium. However, it is interesting to observe the variation in the minimum intensity levels surrounding each individual fibre. This can be seen in the exemplar profiles shown in Fig. 3. The diameter is therefore determined dynamically based on finding the distance from the minimum intensity profile value for both line profiles, which can be considered dual estimates of the radius of each fibre but in orthogonal directions.A further consideration here is regarding the effect of the blurring action of the Point Spread Function (PSF) which will result in an over estimation of the width of a fibre if the limits are taken from the lowest observable points. Therefore in common with e.g. Mets et al. [14], the diameter is taken at the mid-point between the points at which the maximum and minimum are observed. The diameter is thus calculated with

$$
\gamma_{i}=2 \times\left\langle\boldsymbol{x}_{\text {start }}, \boldsymbol{x}_{\mathrm{end}}\right\rangle \times \frac{\left(n_{\mathrm{max}}-n_{\mathrm{min}}\right)}{L}
$$

where $\boldsymbol{x}_{\text {start }}, \boldsymbol{x}_{\text {end }}$ are the start and end points along the Bresenham path but in voxel coordinates with dimensions (e.g. $\mu \mathrm{m})$ respectively; and $n_{\max }-n_{\min }$ and $L$ are the number of voxels along the Bresenham path between the voxels with the maximum and minimum intensities and the total number of voxels along the Bresenham path. The diameter is estimated along both minor axes for every cluster $i$.

A further complication is that the estimate in (9) is possibly dependent on the scale used to derive the candidate fibres through (1). Therefore the mean of the fibre candidates are determined for a given Hessian scale with

$$
\mathrm{E}[\Gamma \mid \eta]=\frac{1}{N_{\eta}} \sum_{\forall i} \gamma_{i}
$$

where $N_{\eta}$ is the number of selected candidate fibres. Each diameter estimate $\gamma_{i}$ will potentially have an error associated with it. Furthermore, the number of detected fibres at any given scale may vary. Any further averaging could potentially benefit by taking into account these factors. Therefore, each diameter estimate $\gamma_{i}$ is considered to be an instance of a Gaussian distributed random variable, i.e. $\Gamma_{\eta} \in \mathcal{N}\left(\mu_{\eta}, \sigma_{\eta}^{2}\right)$ with variance $\sigma_{\eta}^{2}$ and at scale $\eta$ (see (1)). Therefore, an overall diameter estimate, independent of scale can be found with

$$
\hat{\gamma}=\frac{1}{\sum_{\forall \eta} \frac{N_{\eta}}{\sqrt{2 \pi \sigma_{\eta}^{2}}}} \sum_{\forall \eta} \frac{N_{\eta}}{\sqrt{2 \pi \sigma_{\eta}^{2}}}\left(\frac{1}{N_{\eta}} \sum_{\forall i} \gamma_{i}\right) \text {. }
$$

Orientation Distribution and Summarization. The orientation distribution is another important characteristic in many fields of science and engineering when considering the distribution of fibers in a material. It can be characterised purely as a $2 \mathrm{D}$ distribution in terms of the spherical coordinate system consisting the inplane $\theta \in[0 \pi]$ (or polar) angle and out-of-plane angle $\phi \in\left[-\frac{\pi}{2} \frac{+\pi}{2}\right]$. A spherical coordinate system would also require a radius $r$ but this is not usually incorporated into the orientation distribution. For a given vector, the orientation parameters $(r, \theta, \phi)$ can be computed with $r=\sqrt{x^{2}+y^{2}+z^{2}} ; \theta=\tan ^{-1}\left(\frac{y}{x}\right)$; and 


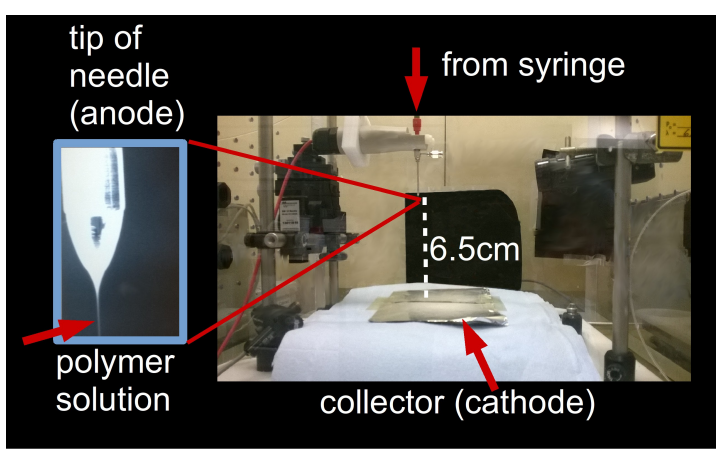

Figure 4: Overview of the experimental setup used to perform electrospinning.

$\phi=\cos ^{-1}\left(\frac{z}{r}\right)$. The angle pair $(\theta, \phi)$ can be approximated via discretization resulting in $(n, m)$ in combination with a distribution $P(n, m)$. The orientation distribution can then be summarised over $P(n, m)$ with a multiscale Shannon's Entropy measure, as shown in [8]

$$
H_{\mathrm{ms}}=\sum_{\forall N} \sum_{\forall M} \sum_{\forall n} \sum_{\forall m} P(n, m) h(n, m)
$$

where the self-information is given by $h(n, m)=$ $-\log _{2}(P(n, m))$ and $N$ and $M$ are the scales over which $(n, m)$ are discretized.

\section{Experimental Details}

Randomly distributed electrospun poly(caprolactone) (PCL) nano-fibre samples were prepared. PCL (80 KDa, 1g) was dissolved in chloroform $(7.5 \mathrm{ml})$ under vigorous stirring, once completely dissolved, methanol $(2.5 \mathrm{ml})$ was added and thoroughly mixed. Solutions were electrospun using the Spraybase device by Profector Life Science Ltd. (Ireland) connected to a custom made capillary nozzle by Rame-hart Instruments Co. (NJ, US) with an internal diameter of 508 $\mu \mathrm{m}$ (Fig. 3). The capillary nozzle was connected to a highvoltage supply $(\max 30 \mathrm{kV})$ operated at $6.5 \mathrm{kV}$ and positioned at $6.5 \mathrm{~cm}$ distance from the collector. Solutions were perfused through the nozzle via a syringe pump (World Precision Instruments, FL, US) ran at $1 \mathrm{ml} / \mathrm{h}$ and fitted with a $5 \mathrm{ml}$ gastight glass syringe (Hamilton). All experiments were carried out at ambient temperature. An overview of the setup of the experiment can be seen in Fig. 4. Fibres were collected, for about $20 \mathrm{~min}$, on a grounded and continuous aluminium foil to achieve a random distribution of the fibres.

The PCL electrospun fibre samples were then scanned with a Zeiss Xradia Versa 510 Microtomography X-Ray Computer Tomography (XCT) system with a voxel resolution of $0.4^{3} \mu \mathrm{m}^{3}$. The resulting scan consisted of $944 \times 975 \times 942$ voxels or, equivalently a $377.6 \times 390 \times 376.8 \mu \mathrm{m}^{3}$ volume. A sub-volume or region-of-interest (see Fig. 1) was then created by cropping the initial scanned volume consisting of $55 \times 100 \times 200$ voxels to simplify subsequent analysis.

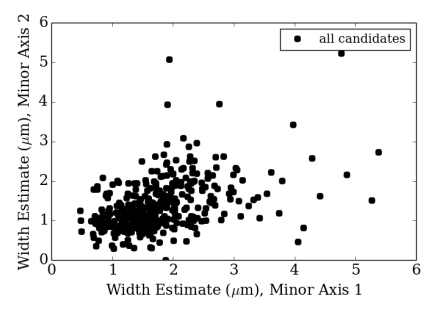

(a) No selection.

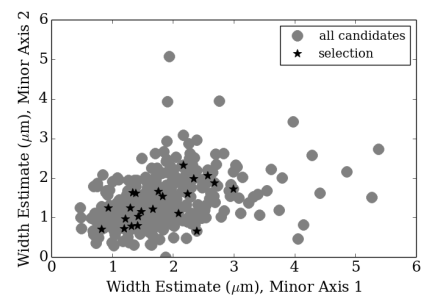

(c) Selection using (6), (7) \& (8).

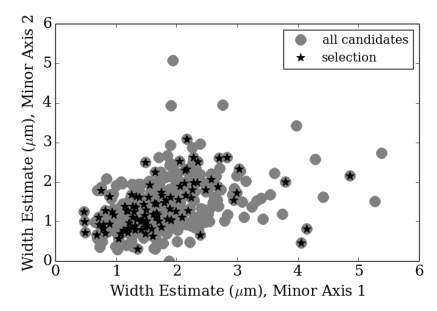

(b) Selection using (6) \& (7).

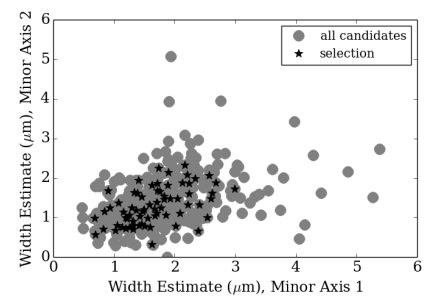

(d) Selection using just (8).
Figure 5: Comparing, via scatter plots, consistency of estimates of the diameter $\gamma$ in (9), which is for a single scale $(\eta)$. The scatter plots using correlation appear to be the most effective at reducing the number of outliers here.

\begin{tabular}{|c|c|c|c|}
\hline selection & axis & $\begin{array}{c}\text { mean } \\
\mu \mathrm{m}\end{array}$ & $\begin{array}{c}\text { st. dev. } \\
\mu \mathrm{m}\end{array}$ \\
\hline \hline none & minor axis 1 & 1.7120 & 0.9223 \\
& minor axis 2 & 1.2698 & 0.6372 \\
& axis mean & 1.4909 & 0.6954 \\
\hline \hline max. \& min. & minor axis 1 & 1.4700 & 0.5001 \\
$(6),(7)$ & minor axis 2 & 1.1443 & 0.4035 \\
& axis mean & 1.3072 & 0.3987 \\
\hline \hline max. \& min. & minor axis 1 & 1.4703 & 0.5673 \\
$\&$ corr. & minor axis 2 & 1.1439 & 0.3633 \\
$(6),(7),(8)$ & axis mean & 1.3071 & 0.4126 \\
\hline \hline corr. & minor axis 1 & 1.4392 & 0.5147 \\
$(8)$ & minor axis 2 & 1.1414 & 0.3511 \\
& axis mean & 1.2903 & 0.3735 \\
\hline
\end{tabular}

Table 1: Results for the fibre diameter estimation process involving differing candidate fibre selections for $\eta=1$.

\section{Results}

Comparisons were made in the effectiveness of rejecting outliers in the fibre diameter estimation process for the two orthogonal axes, as shown in Fig. 5. A table of mean estimated fibre diameters for the different fibre selection techniques is shown in Table 1 . Here it can be seen a range of different diameters have been estimated as a result of the different selection processes.

Scale Selection. It is interesting to note that the results shown above are for a single scale of the Hessian matrix in (1). It would be expected that the diameter estimates might vary at different scales. Therefore the same process of estimating the diameter of the fibres was repeated but using a number of different scales in (1). The resulting diameter estimates can be seen in Fig. 6(a). It can be seen that at greater scales the standard deviation of the estimates increases considerably. Furthermore, as might be expected, at greater scales, the system has detected fibres with greater diameters however, 


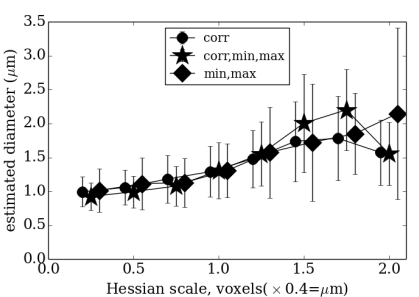

(a)

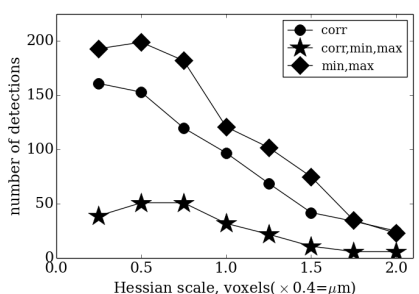

(b)
Figure 6: (a) Mean estimates of $E[\Gamma \mid \eta]$ from (10) as a function of a range of different scale values $\eta$ as used in the computation of the Hessian matrix in (1). The error bars indicate $\pm(\times 1)$ standard deviation of the estimated diameters. (b) Number of detections as a function of scale and selection technique.

\begin{tabular}{|c|c|c|c|}
\hline $\begin{array}{c}\text { scale } \\
\eta(\mu \mathrm{m})\end{array}$ & $\begin{array}{c}\text { mean } \\
\mathrm{E}[\Gamma \mid \eta](\mu \mathrm{m})\end{array}$ & $\begin{array}{c}\text { st. dev. } \\
\sigma(\mu \mathrm{m})\end{array}$ & count \\
\hline \hline 0.25 & 0.993 & 0.221 & 161 \\
0.50 & 1.058 & 0.256 & 153 \\
0.75 & 1.182 & 0.348 & 120 \\
1.00 & 1.290 & 0.373 & 97 \\
1.25 & 1.480 & 0.422 & 69 \\
1.50 & 1.738 & 0.590 & 42 \\
1.75 & 1.783 & 0.621 & 34 \\
2.00 & 1.573 & 0.480 & 25 \\
\hline
\end{tabular}

Table 2: Scale dependent results using correlation only for mean width and error estimation.

because of the large error on these estimates, it could equally mean that the true fibre diameters should actually be much less than the ones estimated using the mean. A further interesting result is in terms of the number of detected fibres as a function of scale and selection technique which can be seen in Fig. 6(b). Here it can be seen that as the scale increases, far fewer fibres are detected, thus reducing the statistical significance in the mean estimation process which is also further hampered with the large standard deviations at greater scales.

Means, standard deviations and fibre detection counts for the three fibre selection processes can be seen in Tables 2, 3 and 4. The estimated means $\mathrm{E}[\Gamma \mid \eta]$ using (10) vary considerably dependent on scale. However, the standard deviations also vary and so do the overall counts of detected fibres. The standard deviation increases for an increasing value of scale. Furthermore the detection count reduces for increasing scale. This means that the mean diameter estimates become less prominent in a technique based on e.g. kernel density esti-

\begin{tabular}{|c|c|c|c|}
\hline $\begin{array}{c}\text { scale } \\
\eta(\mu \mathrm{m})\end{array}$ & $\begin{array}{c}\text { mean } \\
\mathrm{E}[\Gamma \mid \eta](\mu \mathrm{m})\end{array}$ & $\begin{array}{c}\text { st. dev. } \\
\sigma(\mu \mathrm{m})\end{array}$ & count \\
\hline \hline 0.25 & 0.923 & 0.202 & 39 \\
0.50 & 0.995 & 0.233 & 51 \\
0.75 & 1.078 & 0.293 & 51 \\
1.00 & 1.307 & 0.413 & 32 \\
1.25 & 1.553 & 0.471 & 22 \\
1.50 & 2.005 & 0.725 & 11 \\
1.75 & 2.202 & 0.594 & 6 \\
2.00 & 1.555 & 0.467 & 6 \\
\hline
\end{tabular}

Table 3: Scale dependent results using correlation, min. and max. selection for mean width and error estimation.

\begin{tabular}{|c|c|c|c|}
\hline $\begin{array}{c}\text { Scale } \\
\eta(\mu \mathrm{m})\end{array}$ & $\begin{array}{c}\text { mean } \\
\mathrm{E}[\Gamma \mid \eta](\mu \mathrm{m})\end{array}$ & $\begin{array}{c}\text { st. dev. } \\
\sigma(\mu \mathrm{m})\end{array}$ & count \\
\hline \hline 0.25 & 1.014 & 0.321 & 193 \\
0.50 & 1.113 & 0.383 & 199 \\
0.75 & 1.130 & 0.360 & 182 \\
1.00 & 1.307 & 0.399 & 121 \\
1.25 & 1.576 & 0.670 & 102 \\
1.50 & 1.724 & 0.863 & 75 \\
1.75 & 1.851 & 0.601 & 35 \\
2.00 & 2.148 & 1.262 & 23 \\
\hline
\end{tabular}

Table 4: Scale dependent results using min. and max. selection only for mean width and error estimation.

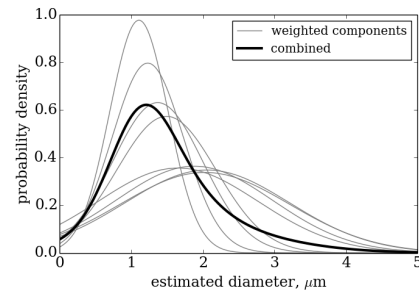

(a) No selection.

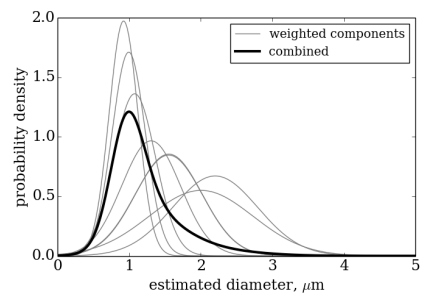

(c) Selection using (6), (7) \& (8).

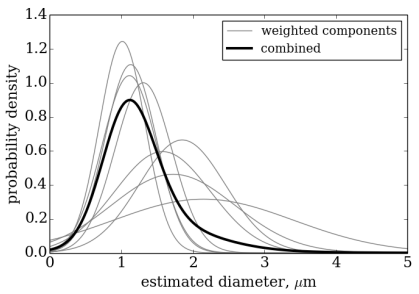

(b) Selection using (6) \& (7).

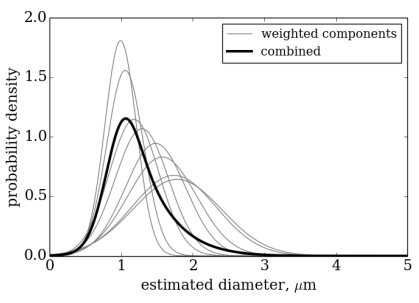

(d) Selection using just (8).
Figure 7: Illustration of the effects of the estimated diameters in a Kernel Density Estimation process, where the estimated parameters at each scale contribute a single Gaussian kernel. The final combined density estimates are also shown.

mation. Kernel density estimates applied to these parameter values, using a Gaussian kernel can be seen in Fig. 7.

The final combined density estimates, also shown in Fig. 7 each have a clearly defined modal point. The overall estimated means and modes can be seen in Table 5 for the 3 different candidate fibre selection techniques. The mean results appear to suggest a mean fibre diameter of $\hat{\gamma}=1.15 \mu \mathrm{m} \pm 0.05 \mu \mathrm{m}$ whereas the mode estimates appear to suggest an overall modal fibre diameter of $1.05 \mu \mathrm{m} \pm 0.05 \mu \mathrm{m}$. These estimates agree well with diameter estimates that have been obtained from Scanning Electron Microscope (SEM) images of the same sample, where smaller fibres, which are beyond the imaging resolution of the XCT, are not included.

\begin{tabular}{|c|c|c|}
\hline selection & $\begin{array}{c}\text { mean } \\
\hat{\gamma}(\mu \mathrm{m})\end{array}$ & $\begin{array}{c}\text { mode } \\
(\mu \mathrm{m})\end{array}$ \\
\hline \hline none & 1.337 & 1.21 \\
corr. & 1.163 & 1.065 \\
corr,min,max & 1.109 & 1.000 \\
min,max & 1.199 & 1.120 \\
\hline
\end{tabular}

Table 5: Overall means and modes for the different candidate fibre selection techniques. The mean, $\hat{\gamma}$ was calculated with (11). The mode was calculated as kernel density estimation. 


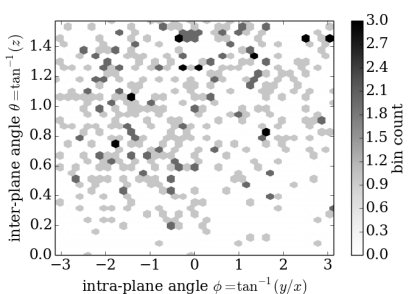

(a) No selection.

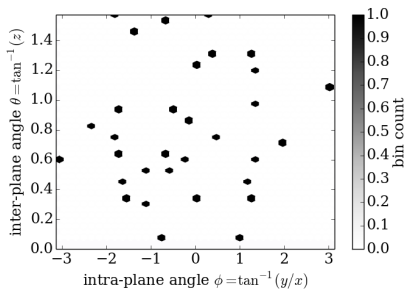

(c) Selection using (6), (7) \& (8).

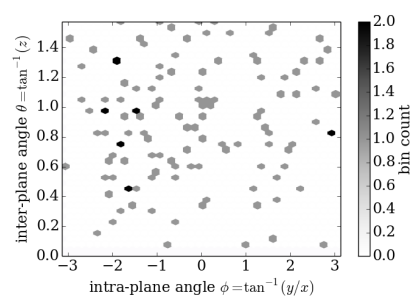

(b) Selection using (6) \& (7).

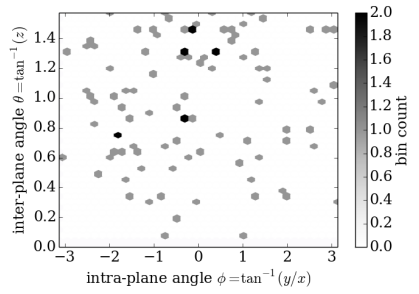

(d) Selection using just (8).
Figure 8: Comparison of orientation distributions when considering differing candidate fibre selection.

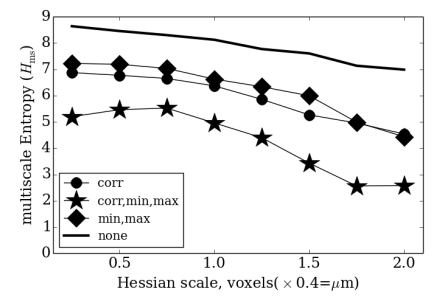

Figure 9: Multiscale Entropy calculations as a function of the Hessian scale and the candidate fibre selection techniques.

Orientation Distribution Results. A comparison of the effects of the different candidate fibre selection criteria on the orientation distribution can be seen in Fig. 8. The orientation distribution can also be conveniently summarised with the multiscale Entropy using (12), as can be seen in Fig. 9. The results in Fig. 9 appear to show a reduction in the entropy with increasing scale in the Hessian which could be due to the reduction in the number of detected fibres. This is somewhat confirmed by the lower entropy estimates for the correlation combined with the min. and max. candidate selection process which has the fewest detections overall, see e.g. Table 3.

\section{Conclusions}

A fibre detection process, along with two main candidate selection processes have been described and investigated. A diameter estimation process has also been proposed that takes into account the partial volume effect. The candidate fibre detection process has also enabled the estimation of the distribution of the orientations of the fibres and further summarization of the orientation distribution using a multiscale Entropy measure. Further work is required to determine the effectiveness of the described techniques in their application to fibres of smaller dimensions.

\section{Acknowledgments}

The authors would like to thank Juliet Massicot for performing some electrospinning work, Marta Peña-Fernandez and Rachna Parwani for the XCT work.

\section{References}

[1] W. Cui, Y. Zhou, and J. Chang, "Electrospun nanofibrous materials for tissue engineering and drug delivery," Sci. Tech. Advanced Materials, vol. 11, no. 1, pp. 1-11, 2010.

[2] Y. Sato, S. Nakajima, N. Shiraga et al., "3D multi-scale line filter for segmentation and visualization of curvilinear structures in medical images," Med. Image Anal., vol. 2(2), pp. 143-168, 1998.

[3] K. Drechsler and C. O. Laura, "Comparison of vesselness functions for multiscale analysis of the liver vasculature," in Proc. 10th IEEE Int. Conf. Info. Tech. Appls. in Biomedicine, Nov 2010, pp. 1-5.

[4] D. Jimenez-Carretero, A. Santos, S. Kerkstra, R. D. Rudyanto, and M. J. Ledesma-Carbayo, "3D Frangi-based lung vessel enhancement filter penalizing airways," in 2013 IEEE 10th International Symposium on Biomedical Imaging, April 2013, pp. 926-929.

[5] R. Rudyanto, S. Kerkstra, E. van Rikxoort et al., "Comparing algorithms for automated vessel segmentation in computed tomography scans of the lung: the VESSEL12 study," Medical Image Analysis, vol. 18, no. 7, pp. 1217 - 1232, 2014.

[6] X. Huang, D. Wen, Y. Zhao, Q. Wang, W. Zhou, and D. Deng, "Skeleton-based tracing of curved fibers from 3d x-ray microtomographic imaging," Results in Physics, vol. 6, pp. $170-$ $177,2016$.

[7] T. Kitrungrotsakul, X.-H. Han, Y. Iwamoto, A. Foruzan, L. Lin, and Y.-W. Chen, "Robust hepatic vessel segmentation using multi deep convolution network," pp. 1013 711-1013 711-6, 2017.

[8] J. Chiverton, O. Ige, S. Barnett, and T. Parry, "Multiscale shannon's entropy modelling of orientation and distance in steel fiber micro-tomography data," IEEE Trans. Image Processing, 2017, accepted.

[9] S. Ahmed, T. Klassen, S. Keyes, M. Daly, D. Jones, M. Mavrogordato, I. Sinclair, and T. Roose, "Imaging the interaction of roots and phosphate fertiliser granules using 4D X-ray tomography," Plant Soil, 2016.

[10] N. Hotaling, K. Bharti, H. Kriel, and C. Simon, "Diameterj: A validated open source nanofiber diameter measurement tool," Biomaterials, vol. 61, pp. 327 - 338, 2015.

[11] J. Chiverton and K. Wells, "Adaptive partial volume classification of MRI data," Physics Med. Biol., vol. 53, no. 20, p. 5577, 2008.

[12] J. F. Barrett and N. Keat, "Artifacts in CT: Recognition and avoidance," RadioGraphics, vol. 24, no. 6, pp. 1679-1691, 2004.

[13] J. Bresenham, "Algorithm for computer control of a digital plotter," IBM Systems J., vol. 4, no. 1, pp. 25-30, 1965.

[14] O. Mets, P. de Jong, B. van Ginneken, H. Gietema, and J. Lammers, "Quantitative computed tomography in COPD: Possibilities and limitations," Lung, vol. 190, pp. 133-145, 2012. 